\title{
Radiopacidade de dentes artificiais para treinamento pré-clínico de endodontia
}

\author{
Artificial tooth radiopacity for preclinical endodontic training
}

\author{
Vanessa Mallmann WESCHENFELDER ${ }^{a}$ (i), Paola Tentardini BAINYa ${ }^{\circ}$,

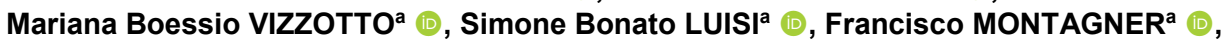 \\ Tiago André Fontoura de MELO ${ }^{\text {* }}$ (1) \\ aUFRGS - Universidade Federal do Rio Grande do Sul, Faculdade de Odontologia, Porto Alegre, RS, Brasil
}

\begin{abstract}
Como citar: Weschenfelder VM, Bainy PT, Vizzotto MB, Luisi SB, Montagner F, Melo TAF. Radiopacidade de dentes artificiais para treinamento pré-clínico de endodontia. Rev Odontol UNESP. 2019;48:e20190053. https://doi.org/10.1590/18072577.05319
\end{abstract}

\begin{abstract}
Resumo
Introdução: Dentes artificiais têm sido utilizados para a prática odontológica e, segundo seus fabricantes, apresentam muita similaridade aos dentes humanos para treinamento. Objetivo: Avaliar a radiopacidade de dentes endodônticos artificiais para uso em atividades de ensino e saber a percepção de docentes quanto à análise radiográfica. Material e método: Trinta réplicas de incisivos centrais superiores de três marcas comerciais (Fábrica de Sorrisos, IM do Brasil e Real Dent) e um incisivo humano foram utilizados. Foram dispostos sobre um sensor radiográfico o dente humano e um dente artificial de cada empresa. As análises de radiopacidade foram feitas no programa Photoshop ${ }^{\circledR}$ em triplicata em quatro regiões distintas: porção central da coroa e nos terços cervical, médio e apical do canal. A percepção quanto à utilização dos dentes como instrumento prático de ensino foi avaliada por meio de um questionário aplicado a oito professores de endodontia. Para análise estatística dos valores de radiopacidade, foi utilizada a ANOVA, seguida do teste de Tukey. Foi empregado um nível de significância de 5\%. Para análise do questionário, foram empregados métodos descritivos e por porcentagem de dados no que se refere à frequência das respostas. Resultado: Houve diferença de radiopacidade entre os diferentes dentes testados em cada região analisada $(p>0,05)$. Na percepção dos professores com relação à utilização dos dentes no ensino, eles colocaram em ordem crescente os seguintes dentes para uso: IM do Brasil, Fábrica de Sorrisos, Real Dent e humano. Conclusão: A radiopacidade dos dentes artificiais foi diferente do humano. 0 dente artificial da Fábrica de Sorrisos apresentou os maiores valores de radiopacidade. Contudo, os professores consideraram ideais para utilização no ensino apenas o dente humano e o dente da Real Dent.
\end{abstract}

Descritores: Educação odontológica; treinamento endodôntico pré-clínico; dentes artificiais; radiopacidade; percepção profissional.

\begin{abstract}
Introduction: Artificial teeth have been used for dental practice which, acoording to their manufactures, bear much similarity to human teeth for training. Objective: To evaluate radiopacity of artificial endodontic teeth for use in teaching activities and to know the perception of teachers regarding radiographic analysis. Material and method: Thirty replicates of upper central incisors of three trade marks (Fábrica de Sorrisos, IM do Brasil and Real Dent) and a human incisor were used. On a radiographic sensor, human tooth and one artificial tooth of each marks were arranged. Radiopacity analyzes were performed using Photoshop ${ }^{\circledR}$ in triplicate in four distinct regions: central portion of the crown and in the cervical, middle and apical thirds of the canal. The perception about the use of teeth as a practical teaching instrument was evaluated through a questionnaire applied to eight teachers of Endodontics. For statistical analysis of the radiopacity values ANOVA was used, followed by the Tukey test. Significance level of $5 \%$. For the analysis of the questionnaire were used descriptive methods and percentage of data regarding the frequency of responses. Result: There was a difference in radiopacity between the different teeth tested, in each region analyzed $(P>0.05)$. In the teachers' perception regarding the use of teeth in teaching, the
\end{abstract}


teachers placed the following teeth for use: IM do Brasil, Fábrica de Sorrisos, Real Dent and human. Conclusion: The radiopacity of the artificial teeth was different from the human teeth. The artificial tooth of the Fábrica de Sorrisos presented the highest values of radiopacity. The teachers considered ideal for use in teaching only the human teeth and the prototype Real Dent.

Descriptors: Dental education; preclinical endodontic training; artificial teeth; radiopacity; professional perception.

\section{INTRODUÇÃO}

As disciplinas pré-clínicas realizadas no curso de Odontologia representam um importante estágio prévio de aprendizagem e treinamento, permitindo que os alunos adquiram habilidades manuais essenciais para o atendimento clínico de pacientes ${ }^{1}$.

o dente humano, por muito tempo, foi o único instrumento para realização desse treinamento pré-clínico. No entanto, hoje, com o risco de infecções cruzadas ${ }^{2}$ e a dificuldade na obtenção dos dentes ${ }^{3}$, estes estão sendo substituídos por réplicas artificiais.

Modelos confeccionados a base de resina têm buscado atender às necessidades do ensino em Odontologia. Entre as inúmeras propriedades que o dente artificial deve apresentar a radiopacidade é um fator importante a ser observado quando utilizado para o treinamento de realização de tratamento endodôntico. Para Nassri et al. ${ }^{4}$, as questões anatômicas e a imagem radiográfica dos dentes artificiais devem ser compatíveis com o dente humano e satisfatórias para que eles sejam utilizados no processo de ensino e aprendizagem.

Dessa forma, o presente estudo tem como objetivo avaliar a radiopacidade dos dentes endodônticos artificiais para uso em atividades endodônticas laboratoriais de ensino e saber a percepção de docentes quanto à análise radiográfica.

\section{MATERIAL E MÉTODO}

O presente estudo foi aprovado pelo Comitê de Pesquisa da Faculdade de Odontologia da UFRGS e pelo Comitê de Ética em Pesquisa da mesma instituição (Protocolo CAAE 01902018.7.0000.5347).

\section{Tipos de Dentes Testados}

Com relação à amostra dentária, foram utilizados 31 incisivos centrais superiores, dos quais 30 foram dentes endodônticos radiopacos artificiais e um humano, obtido na disciplina de Cirurgia e Traumatologia Buco-Maxilo-Faciais da UFRGS.

O incisivo humano foi previamente radiografado para identificação dos seguintes fatores de exclusão: manipulação endodôntica prévia, presença de alterações anatômicas ao longo do canal radicular e fraturas radiculares. 0 dente selecionado foi limpo e passou por um processo de desinfecção, sendo imerso em um pote plástico contendo solução de cloramina a 0,5\% (Seachem Laboratories, Madison, GA, USA) por 48 horas.

Os dentes endodônticos artificiais testados foram obtidos de três empresas comerciais: Fábrica de Sorrisos (Arujá, São Paulo, Brasil), IM do Brasil Ltda. (São Paulo, São Paulo, Brasil) e Real Dent (Rio de Janeiro, Rio de Janeiro, Brasil). Foram adquiridos dez dentes de cada uma das empresas. 


\section{Análise da Radiopacidade}

Sobre um sensor radiográfico oclusal (Digora Soredex, Digora Soredex, Tuusula, Finland), foram dispostos quatro dentes (o dente humano e um dente artificial de cada uma das empresas comerciais analisadas). Com o auxílio de um aparelho de raio X (Timex 70C, Gnatus, Ribeirão Preto, Brasil), acionado a $70 \mathrm{kVp}$ e $8 \mathrm{~mA}$, os conjuntos formados pelos dentes e sensor foram radiografados com tempo de exposição de 0,4 segundo a uma distância foco-filme de $36 \mathrm{~cm}$, conforme especificação $n^{0} 57$ da norma ANSI/ADA ${ }^{5}$. Os dentes foram sempre dispostos com a face vestibular voltada para o cilindro do aparelho de raio X (Figura 1).

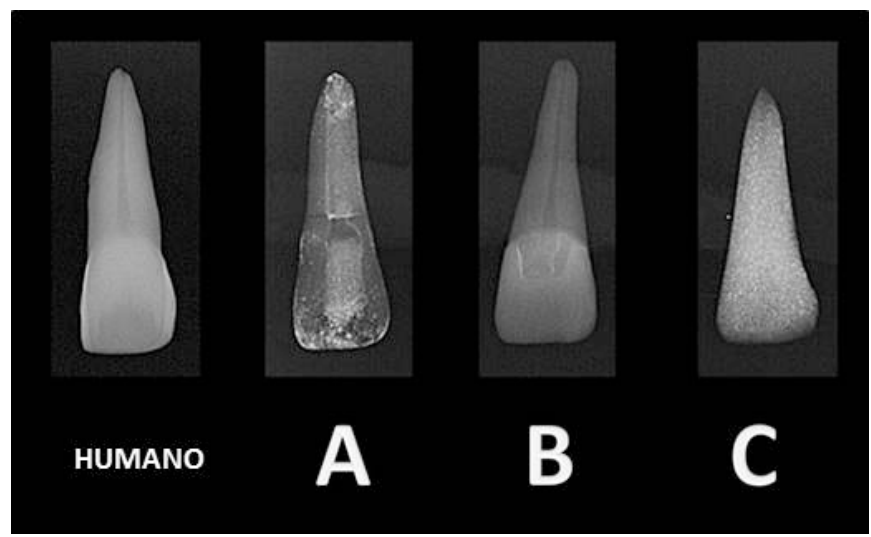

Figura 1. Imagem radiográfica dos quatro dentes testados no estudo ((A) IM do Brasil; (B) Real Dent; (C) Fábrica de Sorrisos).

As imagens digitais foram analisadas no Adobe Photoshop®, software v. 10.0 (Sistemas Adobe, San Jose, CA, EUA). Para análise, um retângulo de tamanho padrão (400 pixels) foi desenhado sobre as áreas de interesse. Os valores de pixel da área selecionada foram medidos usando a ferramenta histograma e registrados.

Em cada elemento dentário, as análises de radiopacidade foram feitas em triplicata em quatro regiões distintas: porção central da coroa dentária e nos terços cervical, médio e apical do canal radicular.

A média dos valores obtidos da triplicata em cada uma das quatro regiões aferidas foi utilizada para comparações estatísticas.

\section{Análise da Percepção da Radiopacidade dos Dentes pelos Professores}

Oito professores de endodontia de instituições de ensino superior no Sul do Brasil foram convidados a participar deste estudo. Cada um deles respondeu a um questionário sobre sua percepção quanto à utilização dos dentes testados como instrumento prático laboratorial de ensino na graduação, com base na análise das imagens radiográficas obtidas. 0 questionário foi estruturado com nove questões fechadas (Apêndice A).

\section{Análise Estatística}

A análise estatística foi realizada com o auxílio do programa GraphPad Prism, versão ${ }^{\circledR}$.

O teste de Shapiro-Wilk foi utilizado para avaliar a normalidade dos dados. Para comparação dos diferentes tipos de dente quanto à radiopacidade, foi utilizada a análise de variância 
(ANOVA), seguida do teste de comparações múltiplas de Tukey, quando necessário. 0 nível de significância adotado foi de $5 \%(\mathrm{p} \leq 0,05)$.

Já para a análise do questionário realizado com professores, foram empregados métodos descritivos e por porcentagem de dados quanto à frequência das respostas.

A hipótese nula do estudo foi a de que não há diferenças estatísticas em relação aos valores da radiopacidade dos diferentes dentes testados.

\section{RESULTADO}

A média e o desvio-padrão dos valores de radiopacidade dos dentes testados no experimento estão expressos na Figura 2.
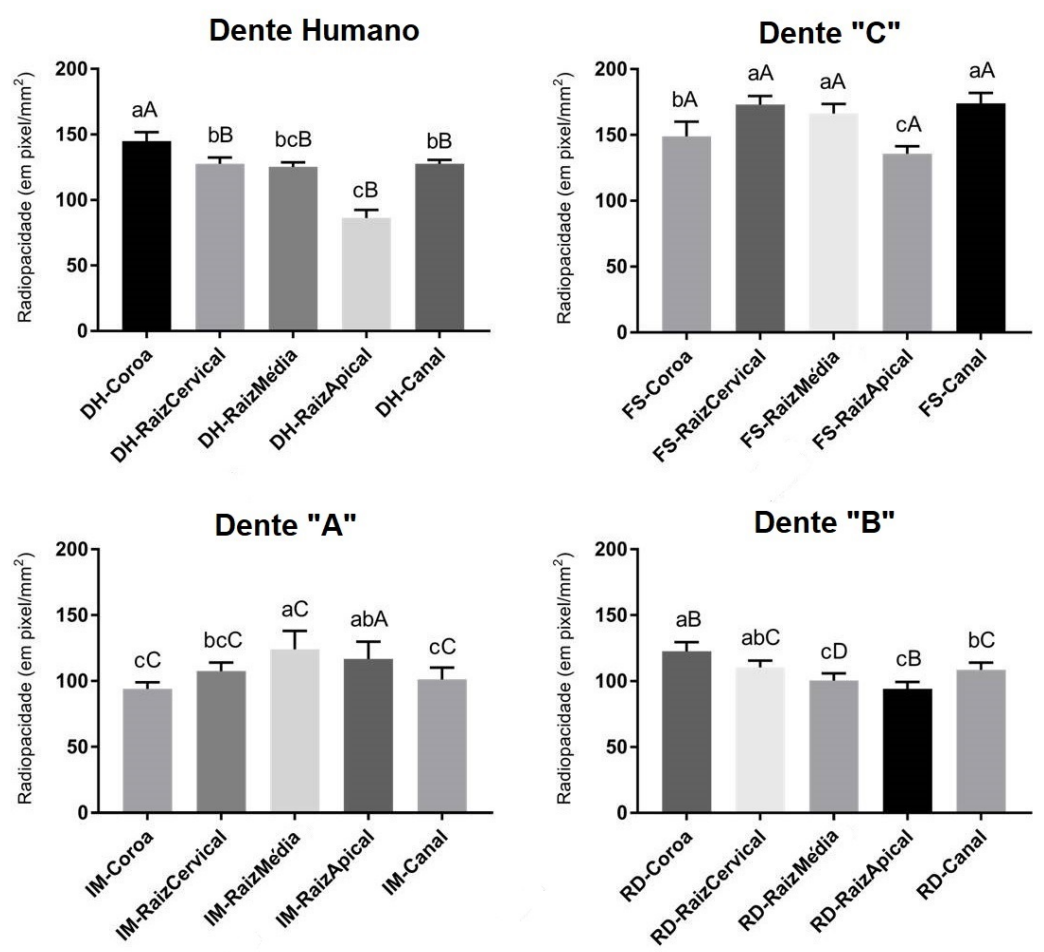

Figura 2. Média e desvio-padrão dos valores de radiopacidade dos dentes avaliados, considerando-se a localização. Letras minúsculas diferentes indicam diferença estatisticamente significativa entre as regiões

de um mesmo dente (ANOVA, teste de Tukey, $p<0,05$ ). Letras maiores diferentes indicam diferença estatisticamente significante entre uma mesma região de diferentes marcas comerciais de dentes (ANOVA, teste de Tukey, $p<0,05$ ).

Com relação às respostas do questionário aplicado aos professores de endodontia, na primeira pergunta eles consideraram boa a qualidade da imagem radiográfica dos dentes humano e artificial "B" (Real Dent). Por outro lado, foram considerados de pior qualidade os dentes "A" (IM do Brasil) e "C" (Fábrica de Sorrisos).

Na segunda pergunta, que apontava se a qualidade da imagem radiográfica visualizada era satisfatória para auxiliar no processo de ensino e aprendizagem de atividades de ensino laboratorial, $100 \%$ dos professores consideraram completamente satisfatórios apenas os dentes humano e artificial "B" (Real Dent).

A porcentagem das respostas dadas pelos professores das questões 3 a 8 está expressa na Tabela 1. 
Tabela 1. Resultados em porcentagem das respostas dadas pelos professores às perguntas 3 a 8 em relação aos diferentes tipos de dente

\begin{tabular}{|c|c|c|c|c|c|c|c|c|c|c|c|c|c|c|c|c|c|c|c|c|}
\hline \multirow{3}{*}{$\begin{array}{c}\begin{array}{c}\text { Opções de } \\
\text { avaliação }\end{array} \\
\text { Item } \\
\text { analisado }\end{array}$} & \multicolumn{4}{|c|}{ Ótima } & \multicolumn{4}{|c|}{ Boa } & \multicolumn{4}{|c|}{ Regular } & \multicolumn{4}{|c|}{ Ruim } & \multicolumn{4}{|c|}{ Péssima } \\
\hline & Dente & IM do & Real & $\begin{array}{c}\text { Fábrica } \\
\text { de }\end{array}$ & Dente & IM do & Real & $\begin{array}{c}\text { Fábrica } \\
\text { de }\end{array}$ & Dente & IM do & Real & $\begin{array}{c}\text { Fábrica } \\
\text { de }\end{array}$ & Dente & IM do & Real & $\begin{array}{c}\text { Fábrica } \\
\text { de }\end{array}$ & Dente & IM do & Real & $\begin{array}{c}\text { Fábrica } \\
\text { de }\end{array}$ \\
\hline & & Brasil & Dent & & humano & Brasil & Dent & & humano & Brasil & Dent & & humano & Brasil & Dent & Sorriso & humano & Brasil & Dent & Sorriso \\
\hline $\begin{array}{c}\text { Coroa } \\
\text { dentária }\end{array}$ & & & $\overline{ }$ & & $12,5 \%$ & & $25 \%$ & & $50 \%$ & & $12,5 \%$ & & $25 \%$ & $12,5 \%$ & $25 \%$ & $12,5 \%$ & $12,5 \%$ & $87,5 \%$ & $37,5 \%$ & $87,5 \%$ \\
\hline $\begin{array}{l}\text { Câmara } \\
\text { pulpar }\end{array}$ & - & & $12,5 \%$ & & $25 \%$ & & $12,5 \%$ & & $25 \%$ & & - & & $37,5 \%$ & & $37,5 \%$ & $12,5 \%$ & $12,5 \%$ & $100 \%$ & $37,5 \%$ & $87,50 \%$ \\
\hline $\begin{array}{c}\text { Canal } \\
\text { radicular }\end{array}$ & $25 \%$ & & $37,5 \%$ & & $62,5 \%$ & & $50 \%$ & - & $12,5 \%$ & & $12,5 \%$ & & - & 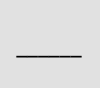 & $\longrightarrow$ & - & 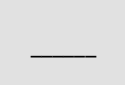 & $100 \%$ & 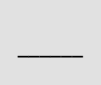 & $100 \%$ \\
\hline $\begin{array}{c}\text { Terço } \\
\text { cervical }\end{array}$ & $12,5 \%$ & 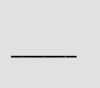 & $12,5 \%$ & - & $50 \%$ & 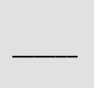 & $37,5 \%$ & 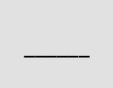 & $12,5 \%$ & - & $12,5 \%$ & 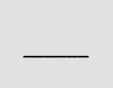 & $25 \%$ & 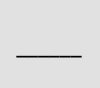 & $37,5 \%$ & $12,5 \%$ & 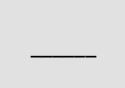 & $100 \%$ & & $87,5 \%$ \\
\hline $\begin{array}{l}\text { Terço } \\
\text { médio }\end{array}$ & $37,5 \%$ & 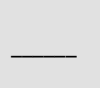 & $37,5 \%$ & - & $50 \%$ & 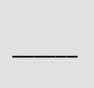 & $50 \%$ & - & $12,5 \%$ & 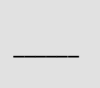 & $12,5 \%$ & - & - & 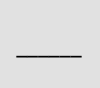 & - & - & ${ }_{-}$ & $100 \%$ & 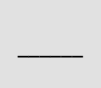 & $100 \%$ \\
\hline $\begin{array}{l}\text { Terço } \\
\text { apical }\end{array}$ & $25 \%$ & 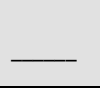 & $37,5 \%$ & 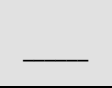 & $62,5 \%$ & - & $50 \%$ & 工 & $12,5 \%$ & 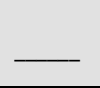 & $12,5 \%$ & 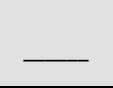 & $\longrightarrow$ & 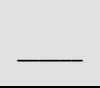 & - & $\longrightarrow$ & $\longrightarrow$ & $100 \%$ & 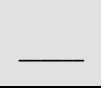 & $100 \%$ \\
\hline
\end{tabular}


Na última pergunta, na qual foi solicitado para os professores colocaram em ordem crescente qual dos dentes eles escolheriam para realizar a atividade prática de pré-clínica, todos indicaram a seguinte sequência: IM do Brasil $\$ Fábrica de Sorrisos $\$ Real Dent

\section{DISCUSSÃO}

Com o surgimento e a utilização de métodos ou recursos alternativos para o ensino endodôntico, o dente artificial tem se tornado uma tendência no processo de ensino e aprendizagem das atividades laboratoriais de graduação. No entanto, várias questões precisam ser discutidas e analisadas quanto à viabilidade de reprodução técnica e ao uso de forma fidedigna dos dentes artificias em substituição aos dentes humanos extraídos.

No estudo, a hipótese nula de que não haveria diferença estatística nos valores de radiopacidade em pixel $/ \mathrm{mm}^{2}$ dos diferentes dentes testados foi rejeitada. Houve diferença de radiopacidade entre os diferentes dentes testados em cada região analisada. Nenhum dos dentes artificiais apresentou valores iguais de radiopacidade aferidos no dente humano em todas as regiões como um todo.

Além disso, quando da comparação dos valores de radiopacidade das diferentes regiões de um mesmo dente artificial, pode-se observar um baixo nível de contraste entre as estruturas anatômicas analisadas. Essa constatação está de acordo com o verificado no estudo de Nassri et al. ${ }^{1}$.

Para Luz et al.3, o baixo valor de radiopacidade apresentado pelos dentes artificiais compromete o desenvolvimento de algumas habilidades técnicas relacionadas à interpretação radiográfica, tornando seu uso limitado em algumas etapas do processo de ensino e aprendizagem. Isso pode ser verificado na comparação entre regiões anatômicas de um mesmo tipo de dente em que não há diferença de radiopacidade, tais como na coroa dentária e no canal radicular do dente artificial da Fábrica de Sorrisos.

A presença de radiopacidade, com diferença de intensidades entre as regiões de um mesmo dente, favorece o entendimento do aluno com relação à execução das etapas endodônticas. Em relação a isso, a radiopacidade apresentada pelos dentes artificiais, agregada à percepção dos professores para o uso desses dentes na prática de ensino, deve ser melhorada, tornando-se a mais próxima possível dos dentes humanos.

$\mathrm{Na}$ análise dos docentes quanto à utilização dos dentes artificias para práticas laboratoriais, o dente humano e o dente artificial da Real Dent foram os mais indicados pelos profissionais para uso nas atividades de ensino. Embora o dente artificial da Fábrica de Sorrisos tenha apresentado valores de radiopacidade próximos ao dente humano, a visualização radiográfica das estruturas dentárias de interesse para realização do tratamento endodôntico não se apresentou nítida. Na prática laboratorial de ensino em graduação, poderia comprometer o entendimento dos procedimentos endodônticos por parte do aluno.

\section{CONCLUSÃO}

A radiopacidade apresentada pelos dentes artificiais foi diferente do dente humano. 0 dente artificial da Fábrica de Sorrisos apresentou os maiores valores de radiopacidade, porém, na percepção dos professores, juntamente ao dente da IM do Brasil, não apresenta características morfológicas em tomadas radiográficas que favoreçam o desenvolvimento de ensino laboratorial. Os professores consideraram ideais para utilização no ensino apenas os dentes humano e artificial da Real Dent. 


\section{AGRADECIMENTOS}

Os autores gostariam de agradecer às empresas Fábrica de Sorrisos e IM do Brasil por fornecerem as amostras para análise.

\section{REFERÊNCIAS}

1. Nassri MRG, Carlik J, Silva CRN, Okagawa RE, Lin S. Critical analysis of artificial teeth for endodontic teaching. J Appl Oral Sci. 2008 Feb;16(1):43-9. http://dx.doi.org/10.1590/S167877572008000100009. PMid:19089288.

2. Tchorz JP, Brandl M, Ganter PA, Karygianni L, Polydorou O, Vach K, et al. Pre-clinical endodontic training with artificial instead of extracted human teeth: does the type of exercise have an influence on clinical endodontic outcomes? Int Endod J. 2015 Sep;48(9):888-93. http://dx.doi.org/10.1111/iej.12385. PMid:25266846.

3. Luz DS, Ourique FS, Scarparo RK, Vier-Pelisser FV, Morgental RD, Waltrick SB, et al. Preparation time and perceptions of Brazilian specialists and dental students regarding simulated root canals for endodontic teaching: a preliminar study. J Dent Educ. 2015 Jan;79(1):56-63. PMid:25576553.

4. Nassri MR, Carlik J, Souza NJA, Montezel JL, Maekawa LE, Oliveira S. Modelo de dentes artificiais com canais simulados para treinamento da Técnica Endodôntica por alunos de graduação. Braz Oral Res. 2005;19(Suppl):21.

5. American National Standard - ANSI. American Dental Association - ADA. (2000). Specification no 57 : endodontic sealing material. Chicago: ANSI/ADA.

\section{CONFLITOS DE INTERESSE}

Os autores declaram não haver conflitos de interesse.

\section{*AUTOR PARA CORRESPONDÊNCIA}

Tiago André Fontoura de Melo, UFRGS - Universidade Federal do Rio Grande do Sul, Faculdade de Odontologia, Departamento de Odontologia Conservadora, Rua Ramiro Barcelos, 2492, Bairro Santana, 90035-003 Porto Alegre - RS, Brasil, e-mail: tiago.melo@ufrgs.br

Recebido: Janeiro 18, 2019

Aprovado: Março 26, 2019 
Apêndice A. Questionário aplicado nos professores de endodontia.

\section{QUESTIONÁRIO DE PESQUISA}

Você está sendo convidado a participar de uma pesquisa que tem como objetivo avaliar a radiopacidade de diferentes dentes endodônticos artificiais para uso em atividades laboratoriais de ensino. Os dados serão mantidos em completo sigilo, sendo utilizados somente para pesquisa e divulgação científica.

1) Como você considera a qualidade da imagem radiográfica do dente?

(1) Péssima (2) Ruim (3) Regular (4) Boa (5) Ótima

2) A imagem visualizada apresenta qualidade satisfatória para auxiliar no processo de ensino e aprendizagem de atividades laboratoriais básicas no ensino pré-clínico da graduação?

(1) Sim (2) Não

3) Como você considera a qualidade da imagem radiográfica do dente analisado no terço cervical?

(1) Péssima (2) Ruim (3) Regular (4) Boa (5) Ótima

4) Como você considera a qualidade da imagem radiográfica do dente analisado no terço médio?

(1) Péssima (2) Ruim (3) Regular (4) Boa (5) Ótima

5) Como você considera a qualidade da imagem radiográfica do dente analisado no terço apical?

(1) Péssima (2) Ruim (3) Regular (4) Boa (5) Ótima

6) Como você considera a qualidade da imagem radiográfica do dente analisado na porção coronária?

(1) Péssima (2) Ruim (3) Regular (4) Boa (5) Ótima

7) A identificação da câmara pulpar na radiografia é:

(1) Péssima (2) Ruim (3) Regular (4) Boa (5) Ótima

8) A identificação do canal radicular na radiografia é:

(1) Péssima (2) Ruim (3) Regular (4) Boa (5) Ótima

9) Enumere, em ordem crescente, qual dos dentes você escolheria para realizar a atividade prática de pré-clínica.

\begin{tabular}{cccc}
\hline Pergunta: & Dente A & Dente B & Dente C \\
\hline Questão 1 & & & \\
Questão 2 & & \\
Questão 3 & & \\
Questão 4 & & \\
Questão 5 & & \\
Questão 6 & & \\
Questão 7 & & \\
Questão 8 & & \\
\hline
\end{tabular}

Questão 9 = 


\begin{tabular}{cccc}
\hline Pergunta: & Dente A & Dente B & Dente C \\
\hline Questão 1 & & \\
Questão 2 & & \\
Questão 3 & & \\
Questão 4 & & \\
Questão 5 & & \\
Questão 6 & & \\
Questão 7 & & \\
Questão 8 & & & \\
\hline
\end{tabular}

Questão 9 =

\begin{tabular}{|c|c|c|c|c|}
\hline Pergunta: & Dente A & Dente B & Dente C & Dente D \\
\hline Questão 1 & & & & \\
\hline Questão 2 & & & & \\
\hline Questão 3 & & & & \\
\hline Questão 4 & & & & \\
\hline Questão 5 & & & & \\
\hline Questão 6 & & & & \\
\hline Questão 7 & & & & \\
\hline Questão 8 & & & & \\
\hline
\end{tabular}

Questão 9 =

\begin{tabular}{cccc}
\hline Pergunta: & Dente A & Dente B & Dente C \\
\hline Questão 1 & & \\
Questão 2 & & \\
Questão 3 & & \\
Questão 4 & & \\
Questão 5 & & \\
Questão 6 & & \\
Questão 7 & & \\
Questão 8 & & \\
\hline
\end{tabular}

Questão 9 =

\begin{tabular}{|c|c|c|c|c|}
\hline Pergunta: & Dente A & Dente B & Dente C & Dente D \\
\hline \multicolumn{5}{|l|}{ Questão 1} \\
\hline \multicolumn{5}{|l|}{ Questão 2} \\
\hline \multicolumn{5}{|l|}{ Questão 3} \\
\hline \multicolumn{5}{|l|}{ Questão 4} \\
\hline \multicolumn{5}{|l|}{ Questão 5} \\
\hline \multicolumn{5}{|l|}{ Questão 6} \\
\hline \multicolumn{5}{|l|}{ Questão 7} \\
\hline Questão 8 & & & & \\
\hline
\end{tabular}

Questão 9 = 


\begin{tabular}{|c|c|c|c|c|}
\hline Pergunta: & Dente A & Dente B & Dente C & Dente D \\
\hline \multicolumn{5}{|l|}{ Questão 1} \\
\hline \multicolumn{5}{|l|}{ Questão 2} \\
\hline \multicolumn{5}{|l|}{ Questão 3} \\
\hline \multicolumn{5}{|l|}{ Questão 4} \\
\hline \multicolumn{5}{|l|}{ Questão 5} \\
\hline \multicolumn{5}{|l|}{ Questão 6} \\
\hline \multicolumn{5}{|l|}{ Questão 7} \\
\hline Questão 8 & & & & \\
\hline
\end{tabular}

Questão 9= 